\title{
Solutions of Partial Differential Equations for Mean Molar Functions
}

\author{
F. E. Wittig \\ Institut für Physikalische Chemie der Universität München \\ Z. Naturforsch. 34a, 99-104 (1979); received November 4, 1978 \\ Dedicated to Prof. Dr. G.-M. Schwab on his 80th birthday
}

\begin{abstract}
The well known formulas for computing the partial molar functions from a given mean molar function are treated as differential equations for computing the mean molar function from any given partial molar function. Solutions do not depend on the number of components, but only on the choice of three indices: the index $d$ of the dependent mole fraction $x_{d}$ to be eliminated prior to any computations, the index $j$ of a pivot mole fraction $x_{j}$ and the index $i$ of the partial molar function $y_{i}$. An arbitrary number of additional mole fractions of the other components safe $x_{d}$ may be linked to the pivot mole fraction $x_{j}$. The simple solution: $y=\left(x_{j}-\delta_{i j}\right) I_{i j}, y_{i}=$ $\left(x_{j}-\delta_{i j}\right)^{2} X_{i j}$ and $X_{i j}=d I_{i j} / \mathrm{d} x_{j}$ holds for an arbitrary number of components, if the $(c-2)$ mole fractions $x_{l}$ safe $x_{d}$ and $x_{j}$ are transformed to new variables found from the auxiliary equations. Three different cases arise if either $i=d, i=j$ or $i \neq d, i \neq j$ is chosen. Formulas for the three sets are provided. As an example a simple interpolation formula for ternary systems is discussed.
\end{abstract}

Previous experimental results on heats of mixing $h^{\mathrm{M}}$ of liquid B-metal binary systems have been evaluated and discussed, using a so called $\xi$-function $[1,2]$

$$
h^{\mathrm{M}}=x_{2}\left(1-x_{2}\right) \xi
$$

as suggested by Wagner [3]. This convenient method however failed in subsequent studies in ternary systems [4]. We had to apply rather intricate computer procedures to find formulas for $h^{\mathrm{M}}$ and the three partial molar heats $h_{i}{ }^{\mathrm{M}}$. Therefore we tried to find simpler methods to process data in ternary systems with simple programmable desk calculators.

A thorough study of pertinent formulas and methods seemed to indicate a missing link in the theory of such functions: Experimental values of excess chemical potentials $\mu_{i}^{\mathrm{E}}=R T \ln \gamma_{i} \quad\left(\gamma_{i}=\right.$ activity coefficient) in binary systems usually are evaluated by integration of the so called GibbsDuhem-equation [3]. In ternary systems integration is possible along particular paths of integration, e.g. $x_{2} / x_{3}=$ constant, as shown by Darken $[3,5,6]$. Another solution by Wagner [3] introduces besides $x_{2}$ a new variable $y=n_{3} /\left(n_{1}+n_{3}\right)$ in the GibbsDuhem-equation. Our previous attempts to find reasonable formulas by trial and error always rendered functions with quotients of mole fractions. Therefore we supposed some hidden reason for the efficiency of such quotients.

Reprint requests to Prof. Dr. Franz E. Wittig, Institut für Physikalische Chemie, Universität München, Theresienstraße 37, D-8000 München 2.
Trouble with such functions seems to have a simple reason: Extensive functions $Y=Y\left(T, p, n_{l}\right)$ are homogeneous functions of the first degree of the $c$ independent mole numbers $n_{l}$ (c number of components). Therefore we get with Euler's equation

$$
\begin{aligned}
Y & =\sum n_{l} y_{l} \quad \text { with the definition } \\
y_{l} & =\left(\partial Y / \partial n_{l}\right)_{T, p, n_{k}}, \quad k \neq l
\end{aligned}
$$

and the more useful differential

$$
\mathrm{d} Y=\sum y_{l} \mathrm{~d} n_{l}
$$

with a short proof. (By partial differentiation to some $n_{k}$ of (1) we get:

$$
\sum_{l} n_{l}\left(\partial y_{l} / \partial n_{k}\right)=0
$$

therefore too

$$
\begin{aligned}
& \sum_{k} \sum_{l} n_{l}\left(\partial y_{l} / \partial n_{k}\right) \cdot \mathrm{d} n_{k}=0 \quad \text { and } \\
& \sum_{l} n_{l} \mathrm{~d} y_{l}=0 .
\end{aligned}
$$

So the differential of (1) renders (2). The differentials $\mathrm{d} y_{l}$ are more versatile, because they can be expanded with any set of appropriate variables of composition.)

In practice, however, the mean molar functions $y=y\left(T, p, x_{l}\right)$ are studied, because the number of independent variables of composition is reduced to $(c-1)$ because of

$$
\sum x_{l}=1 \text { and } \sum \mathrm{d} x_{l}=0 .
$$

In this way labour with experiments and computations is reduced by an order of degree. But this results inevitabily in more trouble with formulas. 
First we have to find how to deal with the new functions and variables. Of course we can derive at once from (1) by division with the sum of number of moles $n$

$$
y=\sum x_{l} y_{l} \text { and } \mathrm{d} y=\sum y_{l} \mathrm{~d} x_{l}
$$

(because from $\sum n_{l} \mathrm{~d} y_{l}=0$, too $\sum x_{l} \mathrm{~d} y_{l}=0$ ).

Such "symmetric" formulas, however, still comprise a dependent mole fraction $x_{d}$, that may be choosen arbitrarily from the $c$ mole fractions in $c$ different ways, and has to be eliminated prior to any computations by

$$
x_{d}=1-\sum^{(d)} x_{l} \quad \text { and } \mathrm{d} x_{d}=-\sum^{(d)} \mathrm{d} x_{l}
$$

by separation of $x_{d}$ in (3) (the symbol $\sum(d)$ indicates, that the term with the index $d$ has been omitted from the sum). From (4) we get

$$
\begin{aligned}
y & =x_{d} y_{d}+\sum^{(d)} x_{l} y_{l} \quad \text { and } \\
\mathrm{d} y & =y \mathrm{~d} x_{d}+\sum^{(d)} y_{l} \mathrm{~d} x_{l}
\end{aligned}
$$

and using (5) finally

$$
\begin{aligned}
y & =y_{d}+\sum^{(d)} x_{l}\left(y_{l}-y_{d}\right) \quad \text { and } \\
\mathrm{d} y & =\sum^{(d)}\left(y_{l}-y_{d}\right) \mathrm{d} x_{l} .
\end{aligned}
$$

Clearly the structure of such "asymmetrical" formulas will be determined by the choice of the index $d$ of the dependent mole fraction $x_{d}$, to be eliminated prior to computations. From (6) we finally get the partial derivatives of the new function $y$ with respect to the new variables $x_{l}$ :

$$
\left(\partial y / \partial x_{l}\right)_{T, p, x_{m}}=y_{l}-y_{d}, \quad m \neq l
$$

only rendering $(c-1)$ equations for the unknown $c$ functions $y_{l}$. Therefore we have to use (6) as additional equation, rendering

$$
y_{d}=y-\sum^{(d)} x_{l}\left(\partial y / \partial x_{l}\right)
$$

as shown by Haase [7, 8].

Clearly the relations between $y$ and the $y_{l}$ are much more intricate than the simple formulas (1) for $Y$ and $y_{l}$ in terms of the mole numbers $n_{l}$.

Sometimes in physics the functions and variables found at first sight are not the most efficients ones with reference to the mathematics involved, as known from theoretical mechanics. Therefore we tried to find other functions and variables, rendering at least one partial molar function as a simple derivative of a function of the molar function.

This problem will be solved by treating the equations for computing the partial molar functions by partial differentiation of the given mean molar function as partial differential equations for the mean molar function, if some partial molar function $y_{i}$ is given. As new function we get the so called integral control function $I_{i j}$ in terms of a pivot mole fraction $x_{j}$ and $(c-2)$ new variables $q_{l j}, r_{l j}$, of $f_{l j}$, depending on the three different possibilities of choosing $i=d, i=j$, or $i \neq d, i \neq j$. The new variables, $q_{l j}$ for quotient, $r_{l j}$ for ratio and $f_{l j}$ for fraction, are quotients of mole fractions, as presumed before. The new functions and variables will be treated as shown with $y$ in terms of the $x$ with formulas (4a), (6), (7) and (8), starting with the differential $\mathrm{d} I_{i j}$. Of course we shall meet the same trouble, because in this case too only $(c-1)$ partial derivatives can be found. This is only a question of the number of independent variables and not of the kind of variables. But we can find one equation rendering a function of a partial molar function as a simple partial derivative of $I_{i j}$ with respect to the pivot mole fraction $x_{j}$.

Later applications are possible without going through the subsequent expositions. Some simple applications and an interpolation formula for ternary system are provided on the last pages. The practical application has to be left to subsequent papers in view of the amount of computations involved. It will take some more papers to deal with the more intricate case of systems with electron transfer [4].

\section{Control Functions}

From (7) and (8) we get a formula for any partial molar function [4]

$$
y_{i}=y-\sum^{(d)}\left(x_{l}-\delta_{i l}\right)\left(\partial y / \partial x_{l}\right)
$$

using the Kronecker-symbol $\delta_{i l}=0$ for $i \neq l$ and $\delta_{i l}=1$ for $i=l$. For any pure component $i$, given by $x_{i}=1$, all other $x_{l}=0$, always $y_{i}=y$ holds. Therefore, any factor in the sum of (9) has to become zero for $x_{i}=1$, all $x_{l}=0$. By this reason we get factors $x_{l}$, but $\left(x_{i}-1\right)$ in $(9)$. In the binary case we get with $x_{j}=x_{1}$ or $x_{j}=x_{2}$ the general formula

$$
y_{i}=y-\left(x_{j}-\delta_{i j}\right)\left(\partial y / \partial x_{j}\right) .
$$

We first tried to get as simple functions and symbols as possible for binary systems. Obviously the structure of the formulas only depend from the indices $i$ and $j$. Therefore the following functions 
and symbols were choosen $[9,10]$

$$
\begin{aligned}
y & =-\left(x_{j}-\delta_{i j}\right) I_{i j} \quad \text { and } \\
y_{i} & =\left(x_{j}-\delta_{i j}\right)^{2} X_{i j} .
\end{aligned}
$$

Putting (11) to (10) the simple partial differential

$$
X_{i j}=\left(\partial I_{i j} / \partial x_{j}\right)
$$

results. $X_{i j}$ and $I_{i j}$ were called control function and integral control function (german: Formfunktion), because such functions carry the information on a particular function in some system, and control the shape of the graphs.

Darken [6] recast (10) to

$$
\frac{y_{i}}{\left(x_{j}-\delta_{i j}\right)^{2}}=\frac{\mathrm{d}}{\mathrm{d} x_{j}}\left(\frac{-y}{\left(x_{j}-\delta_{i j}\right)}\right)
$$

and called the left side function $X_{i j}$ "alpha" and "beta" function, but seemingly did not pay particular attention to the function on the right side. The integral control function $I_{i j}$ is related to the apparent molar functions [9]. Clearly Darken's formula (13) is identical with our formulation (12), but obviously could not be applied to the multicomponent case given by (9).

\section{The Binary Case}

Arranging (10) to

$$
\left(x_{j}-\delta_{i j}\right) y^{\prime}-y=-y_{i}
$$

we get a simple differential equation to compute $y$ from any given $y_{i}$ with the solutions

$$
\begin{aligned}
& y=-\left(x_{j}-\delta_{i j}\right) C \\
& \mathrm{~d} C / \mathrm{d} x_{j}=y_{i} /\left(x_{j}-\delta_{i j}\right)^{2} .
\end{aligned}
$$

Clearly $C=I_{i j}$, and the functions $I_{i j}$ and $X_{i j}$ are solutions of (14).

To get uniform symbols (1) has been replaced by

$$
y^{\mathrm{M}}=-x_{j}\left(x_{j}-1\right) X_{j}
$$

but the control function $X_{j}$ is not a solution of (14) and therefore only useful in binary systems.

\section{The Multicomponent Case}

Arranging (9) we get a partial differential equation

$$
\sum\left(x_{l}-\delta_{i l}\right)\left(\partial y / \partial x_{l}\right)=y-y_{i}
$$

for computing $y$ from a given partial molar funtion $y_{i}$. Lagrange's auxiliary equations, for con- venience written in reverse order

$$
\begin{aligned}
\frac{\mathrm{d} y}{y-y_{i}} & =\frac{\mathrm{d} x_{1}}{x_{1}-\delta_{i 1}}=\frac{\mathrm{d} x_{2}}{x_{2}-\delta_{i 2}}=\cdots \\
& =\frac{\mathrm{d} x_{j}}{x_{j}-\delta_{i j}}=\cdots=\frac{\mathrm{d} x_{c}}{x_{c}-\delta_{i c}}
\end{aligned}
$$

suggest the arbitrary choice of a pivot mole fraction $x_{j}$. (Of course, in (17) and (18) the dependent mole fraction $x_{d}$ is absent. If $d=1, d=2$ or $d=c$, such terms have to be omitted.)

Connecting first $\mathrm{d} y$ and $\mathrm{d} x_{j}$ we arrive at

$$
\left(x_{j}-\delta_{i j}\right) \mathrm{d} y=\left(y-y_{i}\right) \mathrm{d} x_{j}
$$

identical with (14). The solutions (11) and (12) of the binary case hold even with an arbitrary number of components, if the following solutions for the other $(c-2)$ independent mole fractions $x_{l}$ are taken in account. For all other mole fractions $x_{l}$ except $x_{d}$ and $x_{j}$ we find simple proportionality from

$$
\left(x_{l}-\delta_{i l}\right)=k\left(x_{j}-\delta_{i j}\right) .
$$

After fixing the indices $d$ and $j$ of $x_{d}$ and $x_{j}$ obviously three different sets of solutions arise by choice of the index $i$ of the partial molar function $y_{i}$.

\subsection{The $q_{l j}$-set with $i=d$}

Taking $y_{i}$ as $y_{d}$, the Kronecker-symbols in (18) and (19) will vanish rendering from (19)

$$
x_{l}=q_{l j} x_{j} .
$$

The limiting values of the so defined new variables $q_{l j}$ are $q_{l j}=0$ for $x_{l}=0$, but for $x_{j}=0$ the $q_{l j}$ become infinite in any subsystem not containing the component $j$. This may limit the practical use of this set.

\subsection{The $r_{l j}$-set with $i=j$}

Taking $y_{i}$ as $y_{j}$ the Kronecker-symbols $\delta_{j l}$ will vanish, but of course $\delta_{j j}=1$. From (19) follows

$$
x_{l}=r_{l j}\left(1-x_{j}\right)
$$

defining new variables $r_{l j}$ with the convenient limiting values $r_{l j}=0$ for $x_{l}=0$, and $r_{l j}=1$ for $x_{l}=1$.

\subsection{The $f_{l j}$-set with $i \neq d, i \neq j$}

When choosing the index $i$ different from $d$ or $j$, e.g. $i=f$, the corresponding mole fraction $x_{f}$ will appear in (18) or (19) in a bracket $\left(x_{f}-1\right)$, whereas 
all other Kronecker-symbols will vanish. From (19) follows a new set of variables

$$
\left(1-x_{f}\right)=f_{f j} x_{j} \quad \text { and } \quad x_{l}=f_{l j} x_{j} .
$$

The $f_{l j}$ will show the same limiting values as the $q_{l j}$, putting the same limitations on this set.

$$
\mathrm{d} I_{i j}=\frac{\left[y_{d}+\delta_{i j}\left(y_{j}-y_{d}\right)\right] \mathrm{d} x_{j}+\sum^{(d, j)}\left(y_{l}-y_{d}\right)\left[x_{l} \mathrm{~d} x_{j}-\left(x_{j}-\delta_{i j}\right) \mathrm{d} x_{l}\right]}{\left(x_{j}-\delta_{i j}\right)^{2}}, \quad l \neq d, \quad l \neq j .
$$

In the binary case, any $x_{l}=0$ and $\mathrm{d} x_{l}=0$, we get again the solution (11) and (12). With three and more components obviously (24) has to be reduced by judicious choice of new variables to arrive at fairly simple expressions. Any set of new variables can be tested by inserting in (24).

\section{Formulas for the R-set}

According to 3.2 we put $i=j$ and $\delta_{j j}=1$. Inserting (21) and

$$
\mathrm{d} x_{l}=\left(1-x_{j}\right) \mathrm{d} r_{l j}-r_{l j} \mathrm{~d} x_{j}
$$

wet get from (24)

$\mathrm{d} I_{j j}=\frac{y_{j} \mathrm{~d} x_{j}+\left(x_{j}-1\right)^{2} \sum^{(d, j)}\left(y_{l}-y_{d}\right) \mathrm{d} r_{l j}}{\left(x_{j}-1\right)^{2}}$.

Defining the symbols

$X_{j j}=\left(\frac{\partial I_{j j}}{\partial x_{j}}\right)_{r_{l j}}, \quad R_{l j}=\left(\frac{\partial I_{j j}}{\partial r_{l j}}\right)_{x_{j}, r_{k j}}, k \neq l$,

we get the formulas

$$
y_{j}=\left(x_{j}-1\right)^{2} X_{j j} \text { and } y_{l}-y_{d}=R_{l j} .
$$

Unfortunately we get only one simple formula for $y_{j}$, when using the convenient variables $r_{l j}$. For all other partial molar functions we have first to assess $y_{d}$ by inserting (21) and (28) in (6), arranging to

$$
\begin{aligned}
y= & y_{d}\left(1-x_{j}\right)+x_{j} y_{j} \\
& +\sum^{(d, j)} r_{l j} R_{l j}\left(1-x_{j}\right)
\end{aligned}
$$

dividing by $\left(1-x_{j}\right)$, noting (3) and (28) and finally arriving at

$$
y_{d}=I_{j j}-x_{j}\left(1-x_{j}\right) X_{j j}-\sum^{(d, j)} r_{l j} R_{l j} .
$$

Any other partial molar function besides $y_{j}$ and $y_{d}$ is found by

$$
y_{k}=y_{d}+R_{k j}
$$

\section{The Differential $\mathrm{d} I_{i j}$}

Subsequent computations can be reduced by first assessing the differential of $I_{i j}$ (11)

$$
\mathrm{d} I_{i j}=\frac{y \mathrm{~d} x_{j}-\left(x_{j}-\delta_{i j}\right) \mathrm{d} y}{\left(x_{j}-\delta_{i j}\right)^{2}} .
$$

Inserting (6) and arranging we get

or fully

$$
\begin{aligned}
y_{k}=I_{j j} & -x_{j}\left(1-x_{j}\right) X_{j j} \\
& -\sum^{(d, j)}\left(r_{l j}-\delta_{l k}\right) R_{j} .
\end{aligned}
$$

\section{Formulas for the $Q$ - and $F$-set}

Proceeding in the same way we get the following definitions and formulas for use with the less convenient variables $q_{l j}$ and $f_{l j}$ according (20) and (22)

$$
\begin{gathered}
X_{d j}=\left(\frac{\partial I_{d j}}{\partial x_{j}}\right)_{q_{l j}}, \quad Q_{l j}=\left(\frac{\partial I_{d j}}{\partial q_{l j}}\right)_{x_{j}, q_{k j}} k \neq l, \\
y_{d}=x_{j}^{2} X_{d j}, \quad y_{l}=y_{d}-Q_{l j}
\end{gathered}
$$

or fully

$$
\begin{aligned}
y_{l}= & x_{j}{ }^{2} X_{d j}-Q_{l j}, \\
y_{j}= & -I_{d j}-x_{j}\left(1-x_{j}\right) X_{d j} \\
& +\sum^{(d, j)} q_{l j} Q_{l j}
\end{aligned}
$$

and for the $F$-set

$$
\begin{aligned}
X_{f j}= & \left(\frac{\partial I_{f j}}{\partial x_{j}}\right)_{f_{l j}}, \quad F_{f j}=\left(\frac{\partial I_{f j}}{\partial f_{f j}}\right)_{x_{j}, f_{l j}}, \\
F_{l j}= & \left(\frac{\partial I_{f j}}{\partial f_{l j}}\right)_{x_{j}, f_{k j}}, \quad k \neq j, \\
y_{f}= & x_{j}^{2} X_{f j}, \quad y_{d}=x_{j}^{2} X_{f j}-F_{f j}, \\
y_{l}= & x_{j}^{2} X_{f j}-F_{f j}-F_{l j}, \\
y_{j}= & -I_{f j}+x_{j}\left(x_{j}-1\right) X_{f j} \\
& +\left(f_{f j}-1\right) F_{f j}+\sum^{(d, f, j)} f_{l j} F_{l j} .
\end{aligned}
$$

The functions $I_{i j}$ follow from the definition (11):

$$
\begin{aligned}
& y=-\left(x_{j}-1\right) I_{j j}, \quad y=-x_{j} I_{d j}, \\
& y=-x_{j} I_{f j}
\end{aligned}
$$

substituting the new variables from the $R$-, $Q$ - or $F$-set for the $(c-2)$ mole fractions $x_{l}$ besides the eliminated $x_{d}$ and the pivot mole fraction $x_{j}$. 
Formulas for the $Q$ - and $F$-set are simpler, than for the $R$-set. Therefore the $Q$-set may be useful, if the limiting behaviour of the variables $q_{l j}$ for $x_{j}=0$ is of no importance. The somewhat more complicated $F$-set seems to offer no advantages at present.

\section{Examples}

Prior to computations in multicomponent systems some attention should be paid to the judicious choice of the indices $d, i, j$ and $l$. Experimental data of some ternary excess chemical potential $\mu_{i} \mathrm{E}$ may be processed by choosing $i=1,2$ or 3 . But then in view of the advantages of the variables $r_{l j}$ we should prefer $j=i$ to apply the $R$-set. After fixing $i$ and $j=i$, we are free to eliminate one of both remaining mole fractions as the dependent variable $x_{d}$. Then the remaining mole fraction is the $x_{l}$, to be eliminated by $r_{l j}$. Putting e.g. $i=3$, we take $x_{3}$ as pivot mole fraction $x_{j}$. Then we may eliminate $x_{1}=x_{d}$, and take $x_{2}$ as remaining $x_{l}$, to be replaced by $r_{23}=x_{2} /\left(1-x_{3}\right)$. In this way we get the indices $i=j=3, d=1$ and $l=2$. (In systems with $c$ components we get $(c-2)$ different mole fractions $x_{l}$ and therefore as much different indices $l$.) The numbers of indices have to be put into the general equations of the $R$-set, e.g. $X_{33}=\mu_{3} \mathrm{E} /\left(1-x_{3}\right)^{2}$ and $\mathrm{d} I_{33} / \mathrm{d} x_{3}=X_{33}$. Keeping $x_{2} / x_{3}$ constant in Darken's method in ternary systems clearly points to variables of the $Q$-set. In view of (20) we have $x_{l}=x_{2}$ and $x_{j}=x_{3}$. As $x_{2}=q_{23} x_{3}$, this method means to replace $x_{2}$ by $q_{23}$, and to keep $q_{23}$ constant. This means too, to eliminate $x_{1}$ as the $x_{d}$. Wagner's variable

$$
y=n_{3} /\left(n_{1}+n_{3}\right)=x_{3} /\left(1-x_{2}\right)
$$

is related to the $R$-set by choosing $j=2, l=3$ and therefore $d=1$. In view of (21) we get $x_{3}=r_{32}\left(1-x_{2}\right)$ and $y=r_{32}$ in our system.

In ternary systems mean molar functions $y$ can be represented by a power series expansion

$$
y=\sum_{k} \sum_{l} a_{k l} x_{2}^{k} x_{3} l .
$$

Obviously $x_{1}=x_{d}$ or $d=1$. Using (9) the following formulas for the three partial molar functions are found

$$
\begin{aligned}
y_{1}= & \sum_{k} \sum_{l}(1-k-l) a_{k l} x_{2}^{k} x_{3}^{l}, \\
y_{2}= & \sum_{k} \sum_{l}\left[(1-k-l) x_{2} x_{3}+k x_{3}\right] \\
& \cdot a_{k l} x_{2}{ }^{k-1} x_{3}^{l-1}
\end{aligned}
$$

$$
\begin{aligned}
y_{3}= & \sum_{k} \sum_{l}\left[(1-k-l) x_{2} x_{3}+l x_{2}\right] \\
& \cdot a_{k l} x_{2}{ }^{k-1} x_{3}{ }^{l-1} .
\end{aligned}
$$

In this case, the coefficients of $y_{1}$ are simple multiples of the coefficients $a_{k l}$ of $y$.

Choosing $x_{3}$ as pivot mole fraction $x_{j}, j=3$, and preferring the $Q$-set for ease of computation we get

$$
x_{2}=q_{23} x_{3} .
$$

Putting (44) to (40) and dividing by $\left(-x_{3}\right)$ we get regarding (11) and (39)

$$
I_{13}=-\sum_{k} \sum_{l} a_{k l} q_{23}^{k} x_{3}^{k+l-1}
$$

and by differentiation with respect to $x_{3}$ and $q_{23}$ in view of (33)

$$
\begin{aligned}
& X_{13}=\sum_{k} \sum_{l}(1-k-l) a_{k l} q_{23}{ }^{k} x_{3}{ }^{k+l-2}, \\
& Q_{23}=-\sum_{k} \sum_{l} k a_{k l} q_{23}^{k-1} x_{3}^{k+l-1} .
\end{aligned}
$$

Putting $I_{13}, X_{13}$ and $Q_{23}$ in (34) and (35) and finally again substituting $x_{2}$ for $q_{23} x_{3}$ the formulas (41), (42) and (43) are obtained.

\section{A Simple Interpolation Formula for Ternary Systems}

The rather lengthy and tedious computations for assessing the matrix $A_{k l}$ in (40) from experimental data may be considerably reduced by first computing approximate data from the formulas for the binary subsystems using a simple interpolation formula. According to our experience with ternary metallic systems even simple interpolation formulas render about $90 \%$ of the experimental values. As the precision of such data only seldom approaches $1 \%$, the precision of the residue will at best approach $10 \%$. Therefore a simple additional procedure for assessing a matrix for the residue will suffice as a rule.

To assemble interpolation formulas for systems with $c$ components from the formulas for the binary subsystems we have to use some additional indices to indicate different systems. Tentatively, - we have still to gather more experience - the following method is adopted: the numbers of the components are indicated in an additional index. The first number is the index of the mole fraction not used in the formula. In subsystems without the 
component $d$ this will be another mole fraction than $x_{d}$.

In a ternary system $y^{123}$ means the formula for the mean molar function $y$ in terms of $x_{2}$ and $x_{3}$. $y^{23}$ is the formula for the binary system with the components 2 and 3 in terms of $x_{3}$.

Choosing $x_{1}$ as the dependent mole fraction $x_{d}$ and $x_{3}$ as the pivot mole fraction $x_{j}$, and finally putting $x_{2}=r_{23}\left(1-x_{3}\right)$ according to (21) the following simple formula

$$
y^{123}=y^{13}+r_{23}\left(y^{23}-y^{13}\right)+\left(1-x_{3}\right)^{2} y^{12}
$$

allows for linear interpolation between the binary systems (13) and (23), whereas the third system (12) is interpolated by multiplying with $\left(1-x_{3}\right)^{2}$, as done previously by Kohler [10].

In $y^{12}$ the variable $x_{2}$ has to be substituted by $r_{23}$ to stay within the limiting values 0 and 1 of $x_{2}$.

To get the formulas for the three partial molar functions the following procedure is applied:

1. According to (39) we get by division with $\left(1-x_{3}\right)$

$$
I_{33}^{123}=I_{33}^{13}+r_{23}\left(I_{33}^{23}-I_{33}^{13}\right)+\left(1-x_{3}\right) y^{12} .
$$

2. Differentiation with respect to $x_{3}$ and $r_{23}$ renders

$$
\begin{aligned}
& X_{33}^{123}=X_{33}^{13}+r_{23}\left(X_{33}^{23}-X_{33}^{13}\right)-y^{12}, \\
& R_{23}^{123}=I_{33}^{23}-I_{33}^{13}+\left(1-x_{3}\right)\left(y^{12}\right)^{\prime}
\end{aligned}
$$

with $\left(y^{12}\right)^{\prime}=\mathrm{d} y^{12} / \mathrm{d} r_{23}$.

[1] F. E. Wittig, Z. Elektrochem. 63, 327 (1959).

[2] F. E. Wittig and G. Keil, Z. Metallkunde, 54, 576 (1963).

[3] C. Wagner, Thermodynamics of Alloys, AddisonWesley Press, Inc., Cambridge 42, Mass. 1952.

[4] F. E. Wittig, N. Saes and W. Waldherr, Rev. Chim. Minér. 9, 71 (1972).

[5] L. S. Darken, J. Amer. Chem. Soc. 72, 2909 (1950).
3. $y_{1}$ as $y_{d}$ follows from (30) using (49), (50) and (51) as

$$
\begin{aligned}
y_{1}= & I_{33}^{13}-x_{3}\left(1-x_{3}\right)\left[X_{33}^{13}+r_{23}\left(X_{33}^{23}-X_{33}^{13}\right)\right] \\
& +\left(1-x_{3}^{2}\right) y^{12}-r_{23}\left(1-x_{3}\right)\left(y^{12}\right)^{\prime} .
\end{aligned}
$$

4. According to (28) $y_{2}$ is found by simply adding (51) to $y_{1}$

$$
\begin{aligned}
y_{2}= & I_{33}^{23}-x_{3}\left(1-x_{3}\right)\left[X_{33}^{13}+r_{23}\left(X_{33}^{23}-X_{33}^{13}\right)\right] \\
& +\left(1-x_{3}{ }^{2}\right) y^{12}-\left(r_{23}-1\right)\left(1-x_{3}\right)\left(y^{12}\right)^{\prime} .
\end{aligned}
$$

5. $y_{3}$ is found by multiplying (50) with $\left(1-x_{3}\right)^{2}$ according to (28).

Two different checks for such formulas may be applied:

1. Putting the variables equal 0 or 1 and checking the borderline behaviour. Putting e.g. $x_{3}=0(52)$ reduces to $\left(I_{33}^{13}=0\right.$ for $\left.x_{3}=0\right)$

$$
y_{1}=y^{12}-r_{23}\left(\mathrm{~d} y^{12} / \mathrm{d} r_{23}\right)
$$

being the formula for $y_{1}{ }^{12}$ in terms of $r_{23}$.

2. Putting the three partial molar functions in (6) formula (48) will be found.

Applications to evaluations of heats of mixing and of molar free energies of mixing in ternary systems will be presented in subsequent papers. As long as the functions for the binary systems may be represented by power series, the formulas $(48)-(53)$ provide fairly simple rules for assembling matrices for the ternary functions.

[6] L. S. Darken, R. W. Gurry, and M. B. Bever, Physical Chemistry of Metals, McGraw-Hill Book Company, Inc., New York 1953.

[7] R. Haase, Z. Naturforsch. 3a, 285 (1948).

[8] R. Haase, Thermodynamik der Mischphasen, Springer-Verlag, Berlin 1956.

[9] F. E. Wittig, Chemie-Ing. Technik 42, 1037 (1970), 43, 1211 (1971).

[10] F. Kohler, Monatsh. Chem. 91, 738 (1960). 